nungsgemäß nennen, einen Link zur Creative Commons Lizenz beifügen und angeben, ob Änderungen vorgenommen wurden.

Die in diesem Artikel enthaltenen Bilder und sonstiges Drittmaterial unterliegen ebenfalls der genannten Creative Commons Lizenz, sofern sich aus der Abbildungslegende nichts anderes ergibt. Sofern das betreffende Material nicht unter der genannten Creative Commons Lizenz steht und die betreffende Handlung nicht nach gesetzlichen Vorschriften erlaubt ist, ist für die oben aufgeführten Weiterverwendungen des Materials die Einwilligung des jeweiligen Rechteinhabers einzuholen.

Weitere Details zur Lizenz entnehmen Sie bitte der Lizenzinformation auf http://creativecommons.org/ licenses/by/4.0/deed.de.

\title{
Kümmel, Verena: Vergangenheit begraben? Die gestohlenen Leichen Mussolinis und Pétains und der Kampf um die Erinnerung, 370 S., Böhlau, Köln u.a. 2018.
}

\section{Claudia C. Gatzka}

Angenommen: 17. Dezember 2020 / Online publiziert: 5. Januar 2021

(C) Der/die Autor(en) 2021

Wie bestatten Demokratien einen abgesetzten Diktator? Mit dieser Frage schließt Verena Kümmel die politisch gespaltenen Nachkriegsgesellschaften Frankreichs und Italiens auf. Es lag in der Verantwortung der neuen, aus Résistance und Resistenza hervorgegangenen Regierungen, die einstigen Regimeführer Benito Mussolini und Philippe Pétain zu Grabe zu tragen. Die Art der Bestattung, so die Grundannahme Kümmels, symbolisierte die gesellschaftliche Positionierung zum ehemaligen chef de l'État français sowie zum seinerzeitigen Präsidenten der Republik von Salò: Sie rückte die Kollaborateure in ein bestimmtes Verhältnis zu Staat und Nation und präjudizierte die Art, wie ihrer gedacht werden sollte. Da der Maréchal und ,Held von Verdun' ebenso wie der Duce jedoch noch immer von einem beträchtlichen Teil der Bevölkerung verehrt wurden, war die Bestattungsfrage über Jahrzehnte gesellschaftlich umkämpft. Der Diebstahl der Leichen durch neofaschistische Anhänger Mussolinis (1946) beziehungsweise rechtsgerichtete Pétainisten (1973) symbolisier-

C. C. Gatzka $(\bowtie)$

Albert-Ludwigs-Universität Freiburg, Freiburg i. Br., Deutschland

E-Mail: claudia.gatzka@geschichte.uni-freiburg.de 
te die Kritik an der erfolgten Bestattung und macht einen Vergleich der beiden Fälle plausibel.

Mit dem Raub verband sich die Forderung, Mussolinis Gebeine an dessen Witwe zu übergeben beziehungsweise den Leichnam Pétains nach Douaumont zu überführen, wo der Marschall neben den Gefallenen des Ersten Weltkriegs hatte bestattet werden wollen. Kümmel unterzieht die explizite wie implizite symbolische Ausstattung des Bestattungszeremoniells einer konsequent kulturgeschichtlichen, mit den katholischen Traditionen vertrauten und bildgeschichtlich informierten Analyse (Kapitel 2). Gemein war den demokratischen Regierungen der Wille, einen Kontrapunkt zum Personenkult um Mussolini und Pétain zu setzen und keine Pilgerstätten zu stiften, zugleich aber juristisch korrekt zu handeln. Jenseits dessen scheinen vor allem Unterschiede auf: Es gab eben zwei Pétains, jenen von Verdun und jenen von Vichy, und ersterer ließ sich durchaus ehren, wohingegen Mussolini eindeutiger als Diktator signifizierbar war. 1945 von Partisanen exekutiert, dienten Aufbahrung, Leichenschau und Begräbnis in einem anonymen Armengrab ohne Exequien seiner Herabwürdigung, Bestrafung und der Darstellung seiner Schuldigkeit. Demgegenüber billigten die französischen Behörden den 1951 in Haft natürlich verstorbenen Pétain, dessen Todesurteil von Charles de Gaulle aufgehoben worden war, eine respektvolle öffentliche Bestattung auf der Insel Yeu zu, an der Bischöfe und Generäle teilnahmen. Um Pétain konnten Familie und Veteranen trauern, um Mussolini nicht.

Im spannungsgeladenen dritten Kapitel identifiziert Kümmel den Leichendiebstahl als Versuch der Täter und ihrer Drahtzieher, mediale Aufmerksamkeit auf sich und die neofaschistischen und rechten Gruppierungen $\mathrm{zu}$ ziehen, denen sie angehörten, um so den Wahlkampf zu beeinflussen. Die Bemühungen zur raschen Wiederbeschaffung der Gebeine und die damit verbundene Informationspolitik der Regierungen wertet Kümmel als ostentative Demonstration der staatlichen Autorität über die Körper der toten Regimeführer (Kapitel 3). Wie sich die Regierungen jedoch bei der erneuten Bestattung - im Falle Mussolinis nach elfjähriger Verzögerung - auf Kompromisse einließen und so die symbolische Integration Pétains und Mussolinis in die nationale Gedenkgemeinschaft vollzogen, rekonstruiert die Autorin im vierten Kapitel. 1973 war der französische Staat durch einen Kranz symbolisch zu einer Ehrengeste bereit, während die italienische Regierung der Witwe Mussolinis 1957 die Gebeine ihres Mannes übergab und ihr die Regie über die Bestattung in der Familiengruft in Predappio überließ. Der Rückzug des Staates ermöglichte dann auch die Partizipation faschistischer Anhänger an der Bestattungsfeier und löste eine Pilgertätigkeit aus. Kümmel deutet die Regierungsstrategie in beiden Fällen als Signal an die innergesellschaftliche Aussöhnung und arbeitet heraus, inwiefern zivilgesellschaftliche, familiäre und mediale Akteure Druck ausübten. Überdies wird gerade im italienischen Fall die Rolle der katholischen Kirche evident, deren Infrastruktur für die Verstecke der Leiche Mussolinis unabdinglich war.

Die Dissertation kann sich auf eine beträchtliche Fülle an Forschung zum Umgang mit Kollaboration und Bürgerkrieg sowie zum „Körper des Duce“ und zum Nachleben Pétains stützen. Angesichts lückenhafter archivalischer Überlieferung zehrt sie von Erinnerungen aus dem Umfeld der Regimeführer und der am Leichenraub Beteiligten. Durch Relektüre, Plausibilitätsprüfung und bildgeschichtliche Analyse, die auf archiviertes Bildmaterial wie auf Veröffentlichungen in der internationalen 
Presse zurückgreift, fördert sie zum Teil korrigierende Detailbefunde zutage, etwa im Hinblick auf die Motive und Handlungsstrategien der Regierungen und der Leichendiebe. Überaus deutlich wird die unterschiedliche Reinszenierung der Bestattungsereignisse in der Presse, wobei sich transnationale Gemeinsamkeiten in der linken und in der rechten Rezeption feststellen lassen.

Kümmel ist zwar für die Eventualität transnationaler Transfers sensibel, allerdings spiegelt sich die Diachronie der Ereignisse nicht in ihrer Gliederung. Die französischen Behörden konnten bei der Bestattung Pétains den Leichenraub Mussolinis klar vor Augen haben und ihre Strategie entsprechend prophylaktisch ausrichten, jedoch behandelt Kümmel den französischen Fall stets kontrachronologisch vor dem italienischen. Überdies vermag die streng am Bestattungszeremoniell orientierte Darstellung die politikgeschichtlichen Befunde der Arbeit - die Handlungsmacht neofaschistischer und rechter Akteure, für die Vergangenheitspolitik ja immer auch Zukunftspolitik war - nicht systematisch zu präsentieren. Wer aber en detail erfahren will, wie Regierungen, Hinterbliebene und Massenmedien nach 1945 mit toten Diktatoren und verwehrten Staatsbegräbnissen Politik betrieben, wird mit Gewinn zu diesem flüssig geschriebenen Buch greifen.

Funding Open Access funding enabled and organized by Projekt DEAL.

Open Access Dieser Artikel wird unter der Creative Commons Namensnennung 4.0 International Lizenz veröffentlicht, welche die Nutzung, Vervielfältigung, Bearbeitung, Verbreitung und Wiedergabe in jeglichem Medium und Format erlaubt, sofern Sie den/die ursprünglichen Autor(en) und die Quelle ordnungsgemäß nennen, einen Link zur Creative Commons Lizenz beifügen und angeben, ob Änderungen vorgenommen wurden.

Die in diesem Artikel enthaltenen Bilder und sonstiges Drittmaterial unterliegen ebenfalls der genannten Creative Commons Lizenz, sofern sich aus der Abbildungslegende nichts anderes ergibt. Sofern das betreffende Material nicht unter der genannten Creative Commons Lizenz steht und die betreffende Handlung nicht nach gesetzlichen Vorschriften erlaubt ist, ist für die oben aufgeführten Weiterverwendungen des Materials die Einwilligung des jeweiligen Rechteinhabers einzuholen.

Weitere Details zur Lizenz entnehmen Sie bitte der Lizenzinformation auf http://creativecommons.org/ licenses/by/4.0/deed.de. 